\title{
1 Acoustofluidic particle steering
}

2 Zaid Shaglwf', Bjorn Hammarström ${ }^{3}$, Dina Shona Laila ${ }^{2}$, Martyn Hill ${ }^{1}$, Peter Glynne-Jones ${ }^{1 *}$

31 School of Engineering, Faculty of Engineering and Physical Sciences, University of 4 Southampton, SO17 1BJ, UK.

$5{ }^{2}$ School of Mechanical, Aerospace and Automotive, Coventry University, CV1 5FB, UK.

$6{ }^{3}$ Department of Applied Physics, Royal Institute of Technology, AlbaNova University Center,

7 Stockholm, Sweden

8 * Corresponding author: P.Glynne-Jones@soton.ac.uk

9 Keywords: Acoustofluidics, particle manipulation. 


\section{ABSTRACT}

13 Steering micro objects using acoustic radiation forces is challenging for several reasons: resonators tend to create fixed force distributions that depend primarily on device geometry, and even when using switching schemes, the forces are hard to predict a-priori. In this paper an active approach is developed that measures forces from a range of acoustic resonances during manipulation using a computer controlled feedback loop based in MATLAB, with a microscope camera for particle imaging. The arrangement uses a planar resonator where the axial radiation force is used to hold particles within a levitation plane. Manipulation is achieved by summing the levitation frequency with an algorithmically chosen second resonance frequency, which creates lateral forces derived from gradients in the kinetic energy density of the acoustic field. Apart from identifying likely resonances, the system does not require a-priori knowledge of the structure of the acoustic force field created by each resonance. Manipulation of $10 \mu \mathrm{m}$ microbeads is demonstrated over $100 \mathrm{~s} \mu \mathrm{m}$. Manipulation times are of order 10 seconds for paths of $200 \mu \mathrm{m}$ length. The microfluidic device used in this work is a rectangular glass capillary with a $6 \mathrm{~mm}$ wide and $300 \mu \mathrm{m}$ high fluid chamber.

\section{I. INTRODUCTION}

In the recent years, manipulating micro particles and cells using an acoustic radiation forces - acoustofluidics - has found numerous applications in lab-on-chip devices ${ }^{1}$. Potentially useful manipulation operations include bringing cells to specific positions, bringing cells or cells and microbeads together and the control of motile organisms for observation. A comprehensive review and critical analysis of both static and dynamic manipulation strategies and implementation is presented by Drinkwater ${ }^{2}$. We refer the reader to Drinkwater, and present a simpler outline here. Potential applications include re-configurable cell manipulation 
devices for research assays, configurable diagnostic devices, and the tissue engineering. Early devices typically trapped or focussed particles towards locations that were fixed, and dictated by the modes dependent on device geometry ${ }^{3,4}$. A number of approaches have been explored to create flexible manipulation devices that are able to dynamically modulate trapping positions. One option is to switch between resonant modes to create trapping positions that form a continuum of positions, to either translate particles through a range of positions ${ }^{5-8}$ or to create a new fixed manipulation position ${ }^{9}$. An alternative, analogous to optical tweezing, is to use higher frequency systems that form a localised focus and translate the manipulation chamber relative to the transducer ${ }^{10-12}$.

44 The standing wave pattern that determines trapping locations can be considered a superposition of two counter propagating waves. By aligning transducers at an oblique angle 13,14 , utilising transducers that minimise reflections ${ }^{15}$, or relying on absorption ${ }^{16}$, systems can

47 be created that allow each of these components to be independently created with arbitrary phase differences. Thus by modulating the phase difference arbitrary trapping positions can be attained.

Extending this work to circular 2D arrays of transducers, more complex acoustic fields can be created, including acoustic vortices, which contain pressure minima at their centres ${ }^{17,18}$. This

52 is useful since acoustic forces often direct particles towards these locations. Other more complex array based schemes have also been explored in air based resonators ${ }^{19}$, though 54 these go beyond the scope of the current study.

55 An alternative to controlling the pressure node position is to exploit the kinetic energy gradient contribution to the radiation force (see next section), which tends to move particles

57 levitated in a 2D pressure node to regions of maximum acoustic velocity. This movement can 
58 be effected by arrays of transducers ${ }^{20,21}$, or simply by changing the resonance frequency employed as there are often a number of closely spaced resonances around the half-wave resonance of a planar resonator $22-24$.

61 Our work was initially inspired by Armani et al. ${ }^{25}$, who demonstrated the control of particles in a microfluidic system. An algorithm uses camera-based feedback to selectively actuate electro-osmotically driven flows to manipulate multiple particles simultaneously. Our work also has similarity to the manipulation of multiple small objects sitting on a Chladni plate in air as described by Zhou et al. ${ }^{26}$. In this paper, which also uses camera feedback, it was the predictable motion resulting from impacts with the vibrating plate, actuated at various frequencies that provided the particle motion.

In this paper, we present a control system that uses radiation forces (and potentially acoustic streaming too) to manipulate particles levitated in a half-wave resonator. A microscope/camera arrangement images the position of particles, and applies a control algorithm based on a PC to actuate a PZT transducer that can excite a number of different acoustic modes within the resonant manipulation chamber. The frequencies of the modes are determined in advance, but the forces that result from them are assessed and used dynamically without prior knowledge of their magnitude or direction.

\section{BACKGROUND}

As an acoustic wave travels back and forth throughout the chamber of a planar microfluidic device, it creates a standing wave. Due to non-linear effects, there is a small non-zero time average force on particles, the acoustic radiation force ${ }^{27}$. These forces depend on the properties of the particle as well as the shape and amplitude of the acoustic field. The force 
81 the following gradient ${ }^{28}$ :

$83 \quad F=\nabla\left(\left(\frac{3\left(\rho_{p}-\rho_{f}\right)}{\left(2 \rho_{p}+\rho_{f}\right)}\left\langle E_{k i n}\right\rangle-\left(1-\frac{\beta_{p}}{\beta_{f}}\right)\left\langle E_{p o t}\right\rangle\right)\right) V$

with

$$
\begin{aligned}
& E_{p o t}=\frac{\beta_{f}}{2} p^{2} \\
& E_{\text {kin }}=\frac{\rho_{f}}{2} u^{2}
\end{aligned}
$$

84 where $F$ is the time averaged force on a particle of volume $V$, and $E_{k i n}$ and $E_{\text {pot }}$ are the kinetic and potential energy densities respectively, which are functions of acoustic velocity magnitude $u$ and acoustic pressure field $p$, and the brackets $\langle\ldots\rangle$ denote the time average.

87 The particle and fluid densities are $\rho_{p}$ and $\rho_{f}$, while $\beta_{p}$ and $\beta_{f}$ are the particle and fluid compressibilities.

It can be seen that there are two terms that contribute to the force, based on gradients of the kinetic and potential energy densities which (for particles less compressible and denser than the surrounding fluid) direct particles towards acoustic velocity antinodes and pressure nodes respectively (in 2D and 3D standing wave fields, the pressure nodes and velocity anti-nodes are not necessarily coincident ${ }^{29}$ ). In planar resonators it is convenient to decompose the primary acoustic radiation forces into two components, the axial and lateral component. The axial force, which is aligned with the standing wave direction, has contributions from both terms of equation (1), and in a half-wave system tends to levitate particles at the pressure node (which is also approximately coincident with the velocity antinode when the system 
approximates a 1D resonance) at the chamber centre. In the plane of the pressure node, which may undulate ${ }^{30}$, there are no gradients in the potential energy term, and it is only gradients in the kinetic energy which give rise to the lateral component ${ }^{4}$. In this work the levitation position of particles may vary depending on the precise mode that is excited, thus forces from gradients in both terms are likely to act. Additionally, acoustic streaming ${ }^{31}$ could $^{2}$ potentially be present and put to use for manipulation (though in the device presented in this paper, we found that this is a weak effect). Half-wave resonators, as described here, refer to planar resonator structures whose fluid layer height is one half-wavelength. They are typically efficient, and relatively simple to fabricate ${ }^{23}$.

(a)

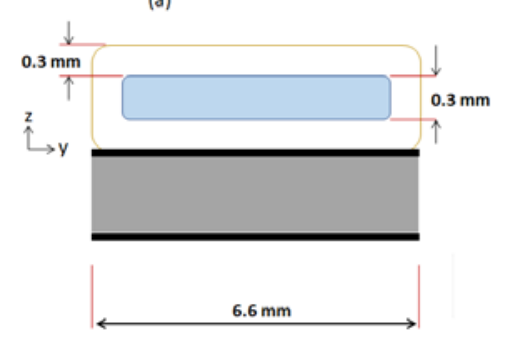

(c) (b)

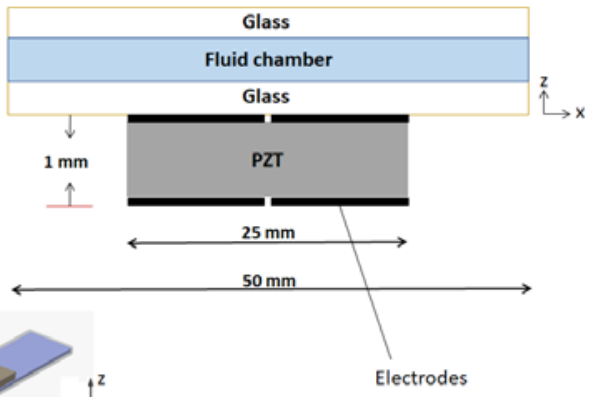
Field of view

Figure 1. Device configuration. (a) Cross-section, (b) Length section and (c) 3D representation. The electrodes have been scored (as seen in the length section) to divide the transducer into two electrically independent regions. The field of view in which manipulation occurs is marked (projected onto the back of the transducer for clarity). Not to scale. 
115 We created a device based on a rectangular glass capillary (ID $0.3 \times 6 \times 50 \mathrm{~mm}$; Vitrocom, NJ, 116 USA). A PZT ( $1 \times 6.6 \times 25 \mathrm{~mm}$, PZ26, Ferroperm) transducer was coupled to the glass capillary 117 using epoxy adhesive (Epoxy 301 Epotek Billerica, MA, USA). The capillary has advantages of mechanical stability, and also lower losses compared to devices with more damping or thicker chamber walls, which will tend to reduce heating effects ${ }^{32,33}$.

The glass capillary was found to create strong lateral modes (and hence forces) across the width of the device (the $y$ coordinate in Figure 1), but relatively less useful forces in the $x$ direction along its length. In order to create modes with forces in the $x$ direction, the transducer was configured with cuts across both the top and bottom electrode surfaces as shown in Figure 1; this divided the transducer into two separate regions, creating asymmetry such that modes created by activating just one of the regions had useful forces in the length direction. It was found that in the area between the two electrode regions there was insufficient levitation forces, so the results in this paper are presented for a manipulation region above the left transducer that is $1 \mathrm{~mm}$ away from the cut and $2.7 \mathrm{~mm}$ from the capillary sidewalls. resonances were found at: $2.48,2.536,2.568,2.814$ and $2.382 \mathrm{MHz}$ (hereafter referred to as resonances or frequencies 1-5). These modes were used for lateral manipulation. The close proximity of these modes does, however, create some sensitivity to temperature variations. The strong half-wavelength resonance found at $2.382 \mathrm{MHz}$ was also used for background levitation, and was applied simultaneously (see section IV) with the manipulating resonances. This mode was applied at lower amplitude for the background

135 levitation, compared to the higher amplitudes used when the lateral forces it produced were required for manipulation. 
137

138

139

140

141

142

Measuring the acoustic pressure amplitude within the resonant cavity is difficult due to the confined space. The acoustic pressure amplitude inside the capillary for a given drive voltage was found by balancing the weight of a $10 \mu \mathrm{m}$ fluorescent polystyrene bead against the acoustic radiation force in the manner described by Spengler et al. ${ }^{34}$. For the levitation mode at $2.382 \mathrm{MHz}$, acoustic pressure was found to be related to drive voltage applied to the PZT by a factor of $31 \mathrm{kPa} / \mathrm{Vpp}$.

\section{SYSTEM CONFIGURATION}

Figure 2 shows the layout of the system hardware. A USB microscope camera (Thorlabs DCC1645C) uses a 10x objective and FITC emission fluorescence filter (Thorlabs MF530-43) to visualise fluorescent microspheres (Fluoresbrite YG $10 \mu \mathrm{m}$, polysciences) that are illuminated by a royal blue 1 W LED (Lumileds, Luxeon Rebel Star, 440-450 $\mathrm{nm}$ ).

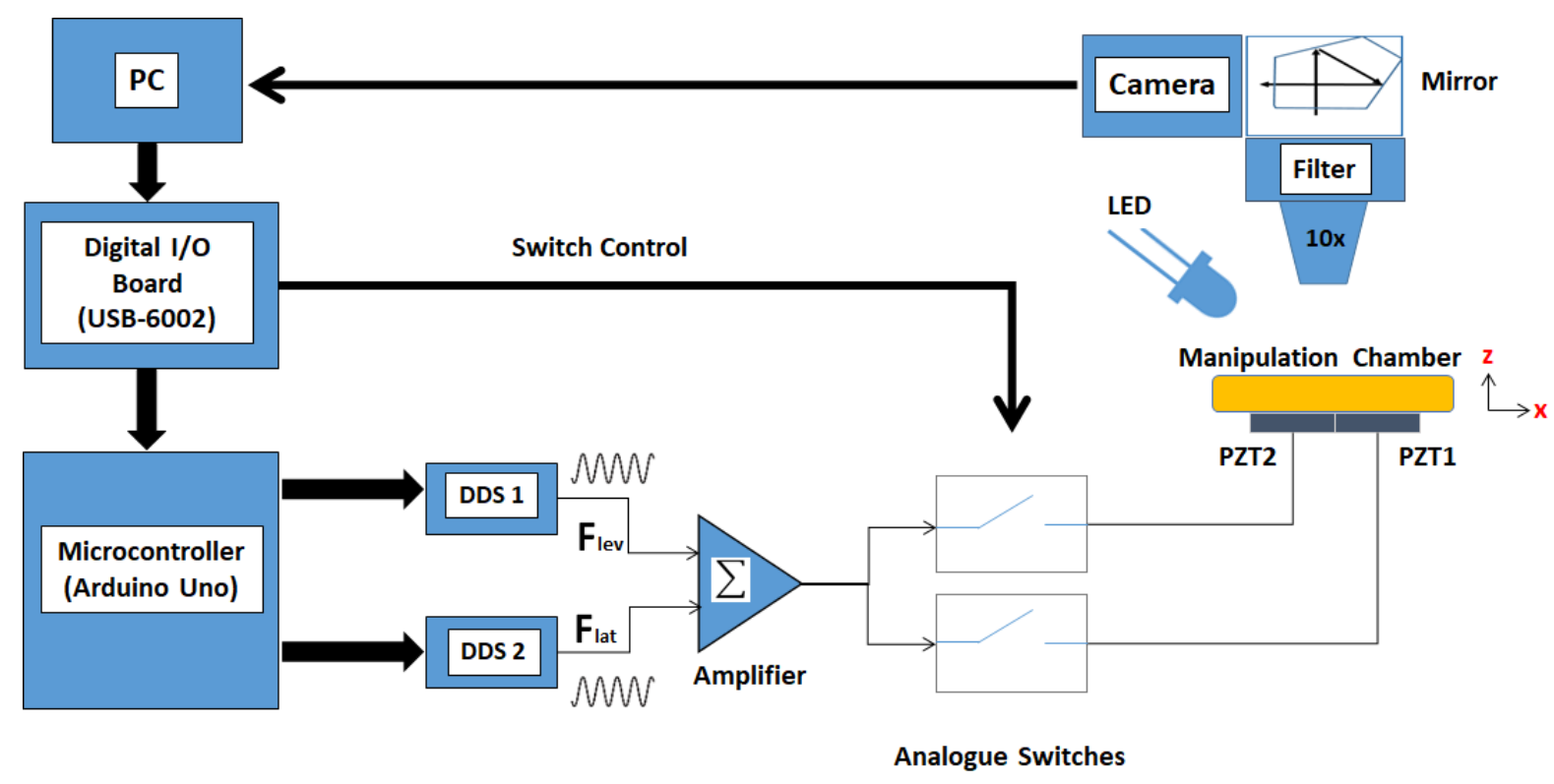

Figure 2. System hardware. A $10 \times$ objective is coupled to a camera via a fluorescence filter and pentaprism mirror cube. It images the microfluidic chamber where ultrasonic resonances are actuated by a PZT transducer with two electrode regions. The control 
152 algorithm based on the PC takes input from the camera and uses an interface board and 153 microcontroller to communicate with DDS boards to synthesise $\mathrm{MHz}$ waveforms to excite resonances. The signals are summed and amplified, then applied to the electrodes under the control of an analogue switch.

157 The camera is connected to a Windows PC that uses MATLAB to execute the control algorithm. Output from the program is routed via a USB digital IO board (NI, USB-6002). This board controls two devices: a) analogue switches (VO14642AT, Vishay Semiconductors) that control which of the transducer regions are activated; and (b) a microcontroller (Arduino UNO) which in turn communicates with two DDS (direct digital synthesis) modules (Analogue devices, AD9850). The Arduinos are preloaded with the required frequencies (determined in advance by examining the impedance spectrum of the transducers and selecting modes that are observed to offer a useful range of forces), and are able to rapidly change the outputs of the DDS modules depending on control signals from the IO board.

It was found that variable latencies between program commands and corresponding changes in USB controller outputs caused errors in the requested pulse length of $\pm 5.3 \mathrm{~ms}$, contributing in part to the errors discussed in Section VII. By keeping pulse lengths to more than $100 \mathrm{~ms}$, these errors were kept reasonably small. The camera exposure time was set to $25 \mathrm{~ms}$, and there was a total delay of 150 ms between issuing a command to capture an image and the data being uploaded and available to the program.

172 One of the DDS modules is used to create a background levitation field $\left(\boldsymbol{F}_{\text {lev }}\right)$ while the second 173 is used to switch between different resonances to carry out the lateral manipulation of the 174 levitated beads $\left(\boldsymbol{F}_{\text {lat }}\right)$. The two signals are combined by a custom summing amplifier (based 
175 on Analogue devices, LT1210). The output amplitudes of the two components are kept fixed

176 at 1.5 and $20 \mathrm{Vpp}$ for the levitation and manipulation frequencies respectively.

177 In order to prevent small fluidic flows occurring during levitation that would interfere with

178 the manipulation, a 6-port valve is used to connect the inlet and outlet ports of the device

179 together after the initial introduction of the sample containing the beads. This equalises

180 hydrostatic pressures and results in drift rates lower than $1 \mu \mathrm{m} / \mathrm{s}$.

181 The camera, pentaprism mirror, fluorescence filter and objective are arranged as shown in

182 Figure 2. Rather than using an expensive microscope, the results presented here were collected from an arrangement that held the imaging components in alignment through a

184 custom laser cut acrylic and 3D printed holder. This arrangement is likely to be more sensitive

185 to vibrations than a conventional microscope, however the steering algorithm was robust to

186 these. (See the next section for how the algorithm deals with this noise in the location measurement).

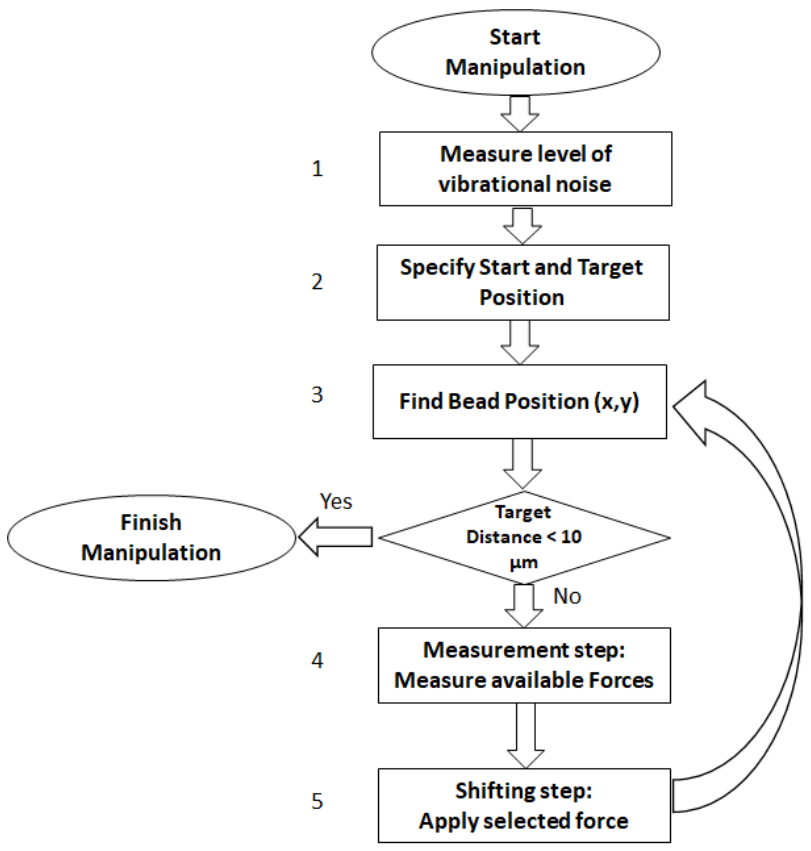


Figure 3. Flow chart showing elements common to both steering algorithms.

192

193

194

195

196

197

198

199

200

201

202

203

204

205

206

207

\section{STEERING ALGORITHM}

The control algorithm is coded in MATLAB, and follows the outline shown in Figure 3 , which is common to both alternative methods discussed here. At the beginning of the manipulation run, a series of 20 images are taken, and the positional measurement accuracy is estimated based on the variation in bead position (resulting primarily from vibration in the system). Typically, this is of order $\pm 1 \mu \mathrm{m}$.

A key feature of the acoustic field is that the forces created tend to vary at a scale of $O(\lambda / 4)$ or longer. Thus, if during a test step the particle travels too far from its initial position there will be some error between the predicted force (based on the initial position) and the actual force created when the resonance is applied starting from the perturbed position. The control algorithms make a linear approximation: that the result of a longer pulse of a resonant frequency will create a particle displacement of a correspondingly longer distance, but in the same direction. By keeping the intended step size small, the effect of the actual non-linearity is reduced. Figure 4 illustrates the measurement process, showing how during the measurement phase the bead makes small movements from its initial one (positions $P_{1-5}$ ) as the resonances are tried in turn. 


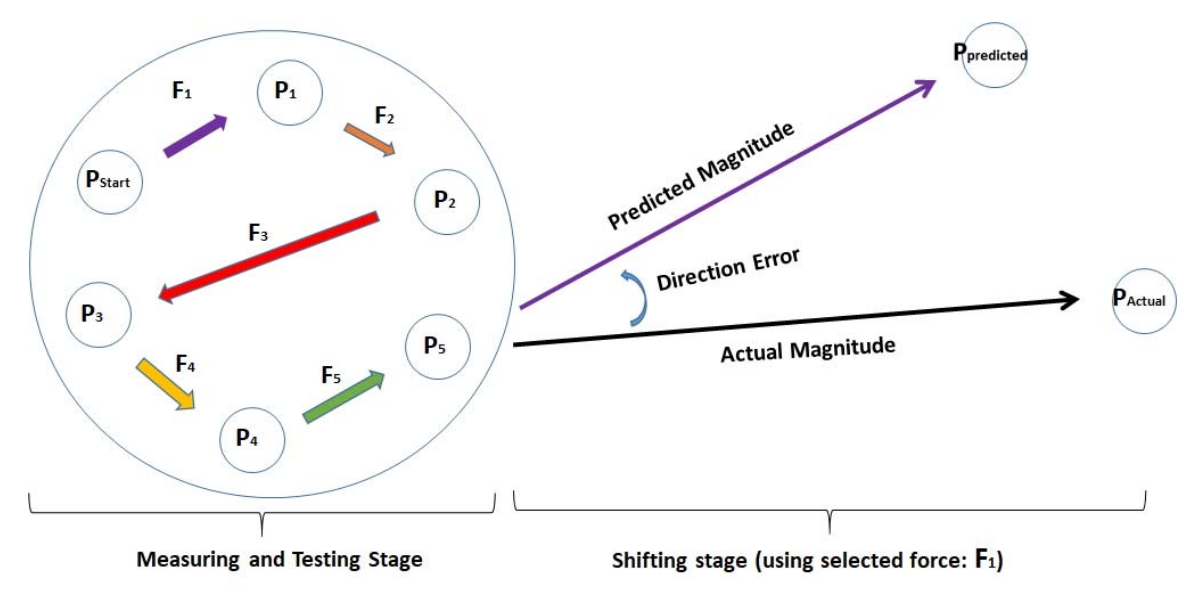

Figure 4. The process of testing and measuring the five manipulation resonances and selecting the one(s) most appropriate to reach the target. The system applies each resonance $\left(F_{1-5}\right)$ in turn and measures the resulting displacement to deduce the force on

212 the particle. The particle moves between five positions $\left(P_{1-5}\right)$; the system then shifts the particle along the manipulation track by applying the selected resonance $\left(F_{\text {selected }}\right)$. There

214 is an error between the predicted and actual particle position after applying the selected

215 force due to measurement errors, timing errors, and variation in acoustic field. Figure not to scale. (Colour online)

218 During measurement phases, the pre-determined set of resonance frequencies are initially tested with a pulse length of $100 \mathrm{~ms}$. Based on the measurements of vibration amplitudes carried out at the beginning of the run and the observed displacement, the predicted error from this source is calculated. If this error is greater than $50 \%$ (i.e. only a small displacement is seen compared to the estimated vibrational noise level) then the measurement is repeated with pulse length calculated to give a $30 \%$ measurement error. If more than 2 secs is required,

224 that resonance is ignored for the step. If this error is lower than $10 \%$ (i.e. the resulting 225 displacement over the measurement step is relatively large) a shorter pulse length is used in 
for the same frequency in the next measurement step, subject to a minimum pulse length of $100 \mathrm{~ms}$. This strikes a balance between testing the available forces with larger pulses of the resonant frequencies (more accurate) versus shorter pulses (which perturb the manipulation track less).

After the measurement phase, the algorithm chooses which resonance, or combination of resonances, is most suitable and applies it to create movement along the manipulation track.

The algorithm continues until either the bead is within $10 \mu \mathrm{m}$ of the target or (as occasionally happens) the bead is lost from the field of view.

Finding the bead's position accurately and quickly is crucial for a successful and rapid manipulation. Speed is achieved by initially searching for particles close to their predicted position by feeding a cropped image to the MATLAB 'imfindcircles()' function which implements a Hough transform. If the particle is not found, a wider region is searched. This approach also helps avoid tracking the wrong particle when more than one is present (although in the current study we typically try to work with only one particle present). Image processing typically takes around $30 \mathrm{~ms}$.

\section{STEERING METHODS}

243 Two alternative implementations are explored here: 1) The combined forces method (CFM)

244 and 2) The Direct method (DM). The two alternatives trade off accuracy through more rigorous measurement (CFM) against speed through less testing (DM).

246 It was found that in the active region there was typically a correspondence between the

247 direction of the lateral forces and which transducer was activated, thus the control algorithm 
chose which transducer to activate based on the direction of the required force. However,

249 since the algorithm measures forces dynamically this simply increased the chance of a suitable

250 force being found without needing to test all possible combinations at each step.

251 Thus, in both approaches, the choice of which transducer to activate (see Figure 2) is given 252 by:

where the angular direction is measured from the $\mathrm{x}$-axis (as defined in Figure 1). The desired direction is the target direction measured from the particle current position. In other words, if the desired direction is in the left or right quadrants, just one transducer is activated (based on the prior knowledge that this tends to produce $x$-directed motion of the correct direction), while if the desired direction is predominantly $y$-directed then both are used.

\section{A. Combined Forces Method (CFM)}

In this approach, the available forces are tested (Figure 3, step 4) after each larger shift step (step 5). After testing, two forces are chosen that when applied sequentially are predicted to produce a net displacement in the direction of the desired target. The algorithm chooses the appropriate forces by finding the nearest pair of clockwise and anticlockwise from the desired direction. Pulse lengths are chosen to create a predicted step of $25 \mu \mathrm{m}$ (except under the special condition b), described in the next paragraph). With the current setup this is achieved by applying each force in turn without imaging to find bead position between them. 
270 a) If the combination of forces required includes using a force that is more than $86^{\circ}$ from the

271 target direction, then just a single force is used, choosing the one closest to the required

272 direction. (The angular threshold is an arbitrary choice, chosen to be slightly less than $90^{\circ}$ )

273 b) If neither of the forces are within $86^{\circ}$ then the closest available force is used with a smaller

274 step size of $12.5 \mu \mathrm{m}$. Although the resulting step is not directly towards the target, there is a

275 chance that a more suitable force will be found after this step.

276 c) If the particle is within $25 \mu \mathrm{m}$ of the target, no more measurement steps are taken, and the

277 pulse length is set to achieve a predicted step of half the remaining distance.

278 Statistical results from a large number of runs are presented in the next section, but we first

279 present an example run to illustrate the process. A bead is manipulated over a $200 \mu \mathrm{m}$ 280 distance, with the conditions at each step along the way shown in Table 1. Figure 5(a) shows 281 the trajectory. The manipulation stops at a distance of $6.43 \mu \mathrm{m}$ from the target (red circle) 282 after a total manipulation time 9.35 seconds. 


\section{Key: Position at end of shift step $\quad$ Measurement steps}

(a)

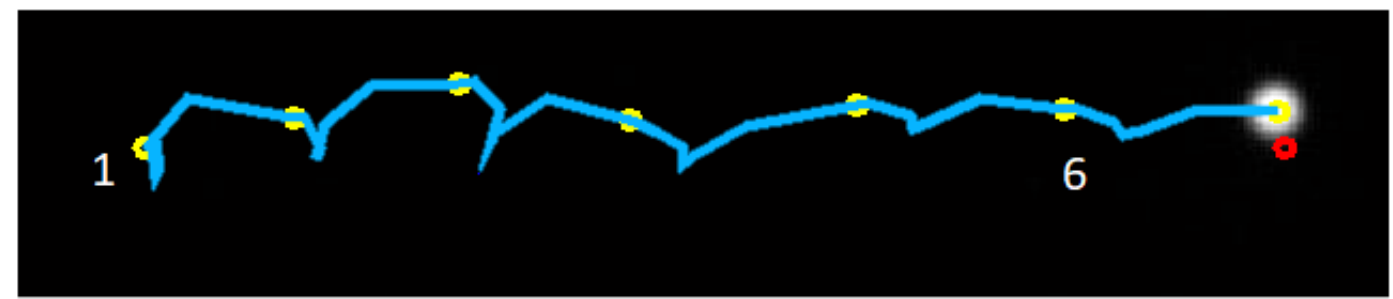

(b)
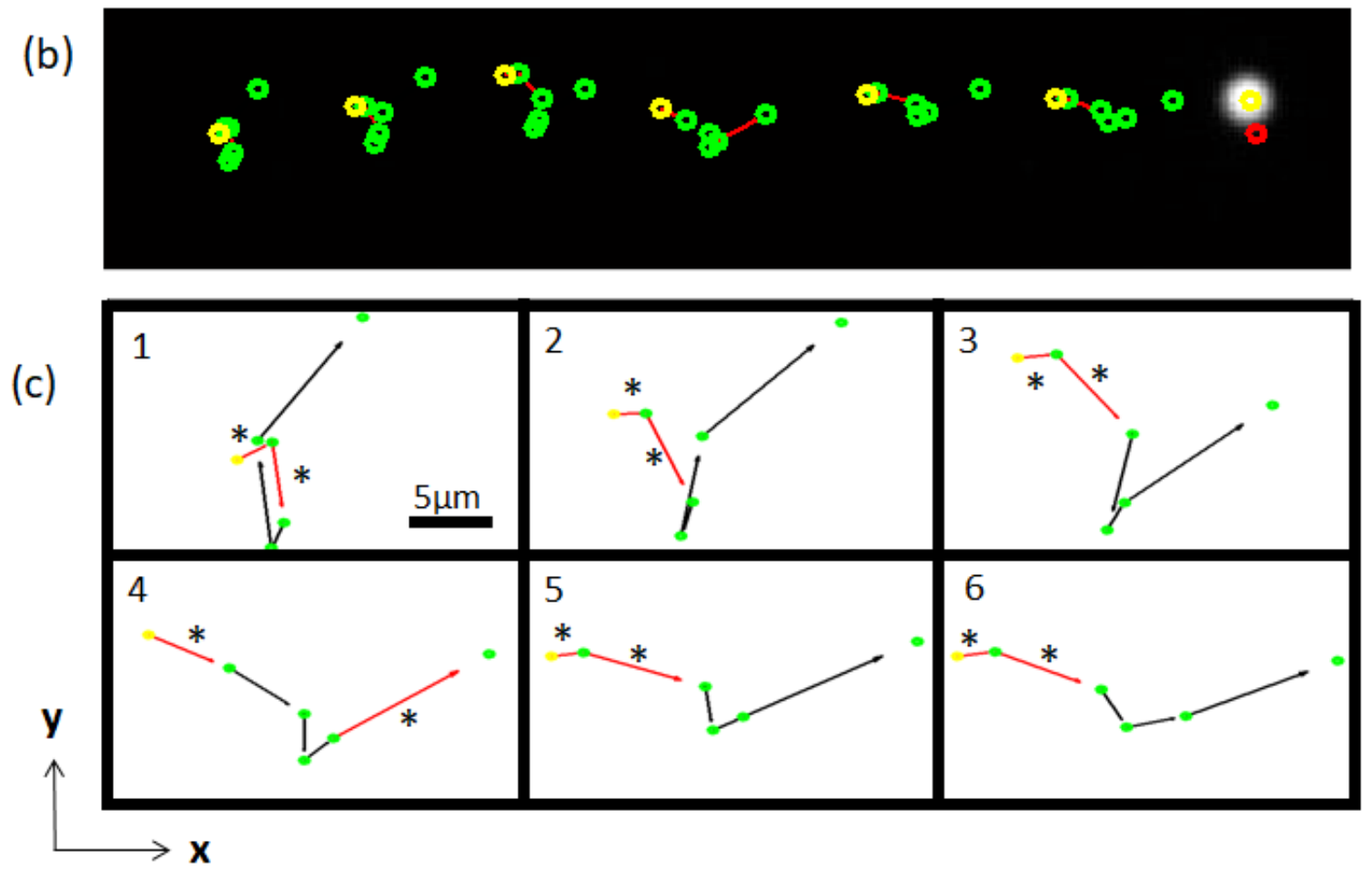

Figure 5. Example steering run with CFM of a levitated $10 \mu \mathrm{m}$ bead over a $200 \mu \mathrm{m}$ distance from left to right towards the target position. $(a, b)$ The trajectory toward the target on the right hand side, including the position at the beginning of each stage, and also the positions (b) after each measurement displacement. (c) A magnified view highlights the two forces that were subsequently chosen for the next shift step with a star $\left(^{*}\right)$. The relationship between the zoomed views, (c), and (a) can be determined from the starting points that they both show. A video of this sequence is shown in the supplementary information ${ }^{36}$. 
293 Table 1: Details of example CFM steering run.

\begin{tabular}{|c|c|c|c|c|c|c|c|c|}
\hline Step & $\begin{array}{l}\text { Required } \\
\text { Direction } \\
\text { at begin } \\
\text { of step } \\
\text { (deg) }\end{array}$ & $\begin{array}{l}\text { Applied } \\
\text { Modes }\end{array}$ & $\begin{array}{c}\text { Distance } \\
\text { moved } \\
\text { during test } \\
\text { phase }(\mu \mathrm{m})\end{array}$ & $\begin{array}{l}\text { Magnitude } \\
\text { error }(\mu \mathrm{m}) \\
\text { (Difference } \\
\text { between } \\
\text { predicted } \\
\text { and actual } \\
\text { magnitude) }\end{array}$ & $\begin{array}{l}\text { Angular } \\
\text { error in } \\
\text { degrees } \\
\text { (Difference } \\
\text { between } \\
\text { predicted } \\
\text { and actual } \\
\text { direction) }\end{array}$ & $\begin{array}{c}\text { Activated } \\
\text { PZT }\end{array}$ & $\begin{array}{l}\text { Time at } \\
\text { begin of } \\
\text { step } \\
\text { (seconds) }\end{array}$ & $\begin{array}{l}\text { Distance to } \\
\text { target }(\mu \mathrm{m})\end{array}$ \\
\hline 1 & 0 & $1 \& 2$ & 11.24 & -5.95 & 9.90 & Right & 0 & 200 \\
\hline 2 & 1.71 & $1 \& 2$ & 14.50 & -9.28 & 3.69 & Right & 1.78 & 173.9 \\
\hline 3 & 4.41 & $1 \& 2$ & 15.32 & -9.64 & 9.74 & Right & 3.54 & 145.1 \\
\hline 4 & 2.29 & $5 \& 1$ & 20.16 & -4.99 & 13.80 & Right & 4.99 & 114.8 \\
\hline 5 & 5.68 & $1 \& 2$ & 21.66 & -10.31 & 1.67 & Right & 7.05 & 75.4 \\
\hline 6 & 9.48 & $1 \& 2$ & 22.55 & -10.03 & -9.88 & Right & 8.13 & 39.3 \\
\hline 7 & & & & & & & 9.35 & 6.4 \\
\hline
\end{tabular}

294 
Table 1 includes a measure of how much displacement is created during the measurement phase (of testing each of the available forces in turn). It can be seen that this distance is significant, however reducing it tends to increase the measurement errors (due to reduced signal to noise, the noise being the result of unwanted vibrations). See section $V$ for discussion of this.

As shown in Figure 5 (b) and (c), the steering system measures the forces available at each step. For example, for the first step, the available five forces have directions: $332.3,80.2$, $114.5,263.5$ and 309.3 degrees respectively. As the required direction towards the target for this step is 0 degrees, the steering algorithm chooses forces $1 \& 2$, the nearest to this direction in the anticlockwise and clockwise directions and applies them sequentially, for 947 and 213 ms respectively, aiming to achieve a step of $25 \mu \mathrm{m}$ in the required direction (the total step time is longer than this as time is required for the imaging). Note that no image is taken by the system between these two actuations. This is repeated at each step until the bead is within $10 \mu \mathrm{m}$ of the target. The advantage of combining forces in this method are clearer in steps (such as step 1), when the available forces only have small components in the desired direction.

\section{B. Direct method (DM)}

In order to create faster manipulation times and to take advantage of the knowledge that acoustic forces only vary over distances of order $\lambda / 4$ or greater, this method measures the available forces less often. After the initial measurement step, the force that is closest to the desired direction is chosen. If the selected force is within 0.5 radians ( 28.6 degrees) of the required direction then an actuation pulse is applied for a time that is calculated to give a 25 $\mu \mathrm{m}$ displacement. The value of this angular threshold was an arbitrary choice that gave 
reasonable results; further study could investigate optimising it. However, if the difference is more than this then the system aims for only $12.5 \mu \mathrm{m}$ per step. This is in order to allow for

321 the possibility of more suitable forces in subsequent steps. The requested distance is also

322 reduced to half the target distance if the bead is closer than $50 \mu \mathrm{m}$ to the target (and at this

323 range, further measurements are not taken, relying instead on the last measurement phase).

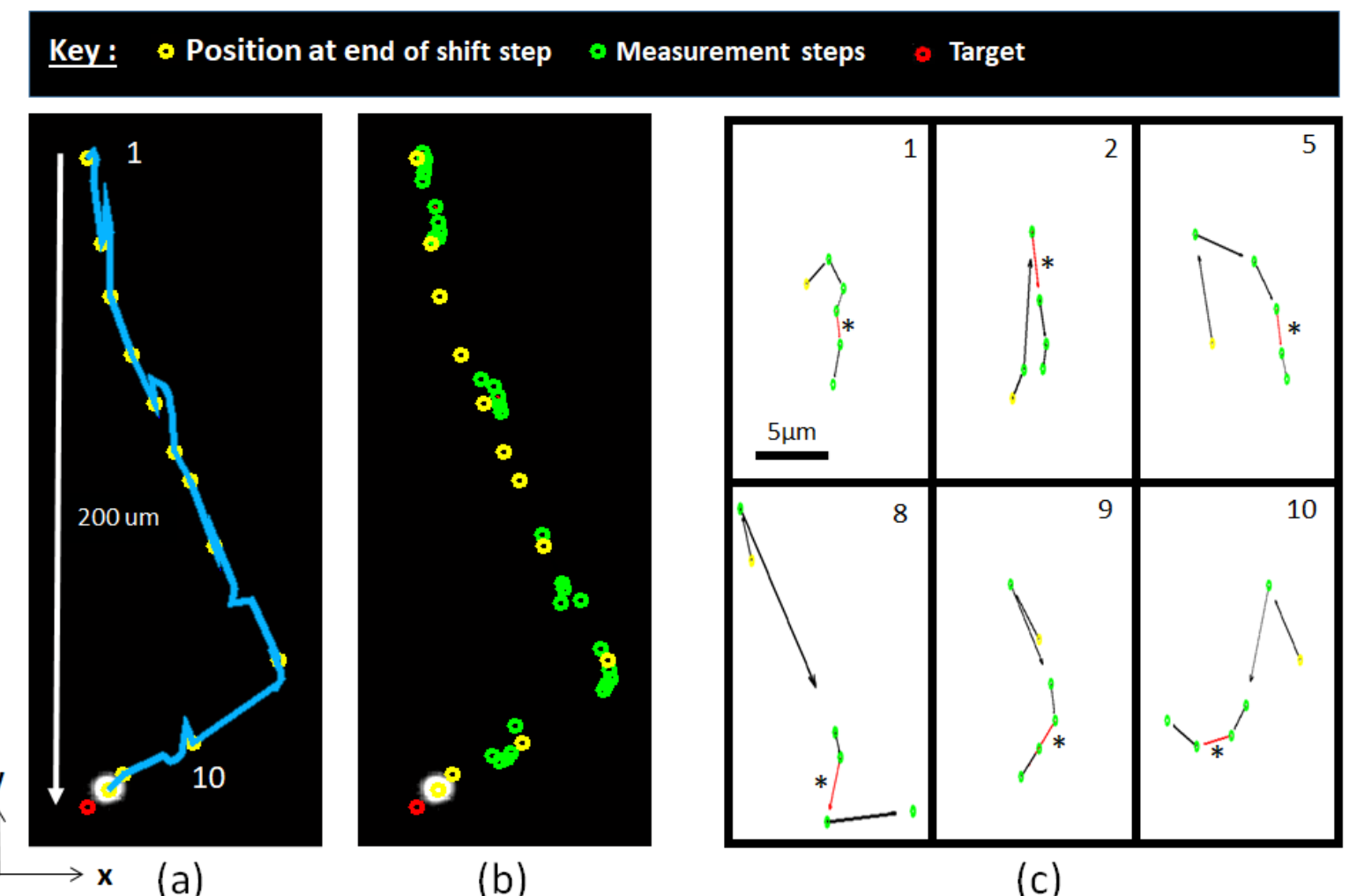

Figure 6. Example steering run for the direct method (DM) of a levitated $10 \mu \mathrm{m}$ bead over a toward the target, including the position at the beginning of each stage, and also the positions (b) after each measurement displacement. (c) A magnified view highlights the forces that were subsequently chosen for the next shift step with a $\operatorname{star}\left({ }^{*}\right)$. A video of this sequence is shown in the supplementary information ${ }^{37}$. (Colour online) 
332 In subsequent steps, the forces are not measured again unless the distance to the last

333 measurement point is more than $25 \mu \mathrm{m}$. Instead, the algorithm stores the values previously

334 measured in a table and uses these. After each movement step, the entry in the table for the

335 force that was used is updated to reflect the actual force observed acting over the step. This

336 takes account of the higher accuracy of the measurement given the larger displacement

337 compared to the measurement phase, and also makes allowance for the force changing with

338 position and changing over time due to any drifts associated with heating effects.

339 An example manipulation is shown in Figure 6, with details shown in Error! Reference source

340 not found.. It can be seen that measurements are only made in 6 of the 11 steps. A further

341 example of a bead being manipulated along a square trajectory is shown in a video provided

342 in the supplementary information ${ }^{35}$.

344 Table 2: Details of example DM steering run.

\begin{tabular}{|c|c|c|c|c|c|c|c|c|c|c|}
\hline Step & $\begin{array}{l}\text { Required } \\
\text { Direction } \\
\text { (deg) }\end{array}$ & $\begin{array}{l}\text { Applied } \\
\text { Mode }\end{array}$ & $\begin{array}{c}\text { Distance } \\
\text { moved } \\
\text { during } \\
\text { test } \\
\text { phase } \\
(\mu \mathrm{m})\end{array}$ & $\begin{array}{l}\text { Magnitude } \\
\text { error }(\mu \mathrm{m}) \\
\text { (Difference } \\
\text { between } \\
\text { predicted } \\
\text { and actual } \\
\text { magnitude) }\end{array}$ & $\begin{array}{l}\text { Intended } \\
\text { shifting } \\
\text { magnitude } \\
\qquad(\mu \mathrm{m})\end{array}$ & $\begin{array}{l}\text { Angular } \\
\text { error (deg) } \\
\text { (Difference } \\
\text { between } \\
\text { predicted } \\
\text { and actual } \\
\text { direction) }\end{array}$ & $\begin{array}{l}\text { Difference } \\
\text { between } \\
\text { selected } \\
\text { and } \\
\text { required } \\
\text { direction } \\
\text { (deg) }\end{array}$ & $\begin{array}{c}\text { Activated } \\
\text { PZT }\end{array}$ & $\begin{array}{c}\text { Time } \\
\text { (seconds) }\end{array}$ & $\begin{array}{c}\text { Distance } \\
\text { to target } \\
(\mu \mathrm{m})\end{array}$ \\
\hline 1 & 90.00 & 4 & 7.06 & -5.28 & 25 & -1.15 & 6.83 & BOTH & 0 & 200 \\
\hline 2 & 91.49 & 3 & 2.88 & -7.00 & 25 & 0.08 & 3.15 & BOTH & 0.63 & 173.7 \\
\hline 3 & 92.57 & 5 & No Test & -5.61 & 25 & -27.24 & 4.81 & BOTH & 1.64 & 157.82 \\
\hline 4 & 95.60 & 4 & No Test & -8.45 & 25 & -16.51 & 14.51 & BOTH & 2.14 & 140.1 \\
\hline
\end{tabular}




\begin{tabular}{|c|l|c|c|c|c|c|c|c|c|c|}
\hline 5 & 99.48 & 4 & 5.63 & -12.61 & 25 & 1.98 & 16.46 & BOTH & 2.42 & 126.2 \\
\hline 6 & 103.80 & 5 & No Test & -15.09 & 25 & -17.97 & 24.87 & BOTH & 3.12 & 113.0 \\
\hline 7 & 107.45 & 3 & No Test & 9.03 & 12.5 & 1.57 & 39.24 & BOTH & 3.60 & 105.9 \\
\hline 8 & 115.88 & 4 & 20.28 & -4.70 & 25 & -31.25 & 18.84 & BOTH & 4.16 & 89.8 \\
\hline 9 & 142.29 & 4 & 7.37 & 6.70 & 25 & 17.79 & 14.54 & LEFT & 5.65 & 74.1 \\
\hline 10 & 148.33 & 4 & 9.89 & -5.55 & 19.07 & -5.61 & 13.47 & LEFT & 7.12 & 38.2 \\
\hline 11 & 136.54 & 3 & No Test & -0.97 & 7.63 & 14.32 & 19.97 & LEFT & 7.70 & 15.3 \\
\hline 12 & & & & & & & & & & \\
\hline
\end{tabular}

345

346 


\section{COMPARISON AND DISCUSSION}

350 A series of experiments were performed aiming to steer beads along the sides of a square

351 shape of side $200 \mu \mathrm{m}$. In each case a random start position was chosen, and the bead directed

352 towards a target point on the next corner of the square. This continued until either the

353 original position was again reached or the manipulation failed. Each traverse of the side of a

354 square is called a "leg" here. Both algorithms were examined, with results presented here for

35530 CFM legs and 95 DM legs.

356 The distribution of the number of steps required to reach the target and the proportion of

357 successful legs is shown in Figure 7. Legs are allowed to continue until either the target is

358 reached ("success") or the particle is lost by the tracking algorithm ("fail" - typically due to

359 the particle leaving the field of view). The CFM typically reaches the target in fewer steps that

360 the DM: $80 \%$ of successful legs are completed in 0-30 steps for the CFM compared to only

$36169 \%$ for the DM. The difference between the number of failures in each case is not significant,

362 as this relates mainly to legs when the bead was lost by virtue of having started close to the

363 edge of the field of view or an additional bead entered the field of view and confused the 364 algorithm. 


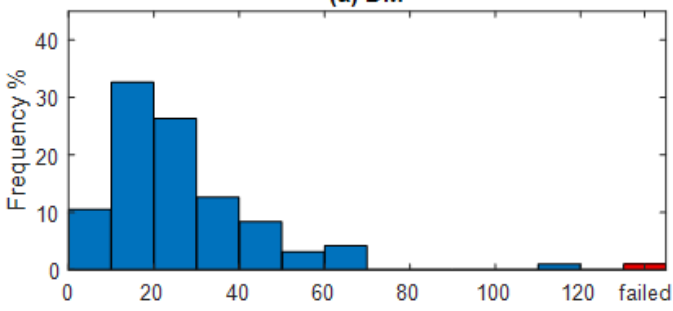

(B) CFM

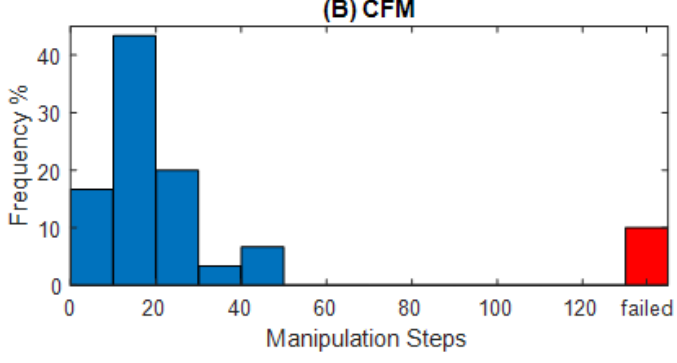

366 Figure 7. Histograms showing the number of steps to a successful manipulation for a) the 367 combined forces method (CFM) and b) Direct method (DM). The algorithm continued in all

368 cases until either the bead came within the $10 \mu \mathrm{m}$ target distance (successful legs) or the 369 bead was lost by the tracking code (failed legs). It can be seen that on average the CFM is 370 more effective at reaching the target in fewer steps than the DM. The plots represent data from 30 and 95 legs respectively.

373 The error between the predicted (as calculated from measurement) and the actual bead 374 displacements during the shifting stage is shown in Figure 8, where the positive values 375 indicate the actual (direction/magnitude) are greater than predicted values. 

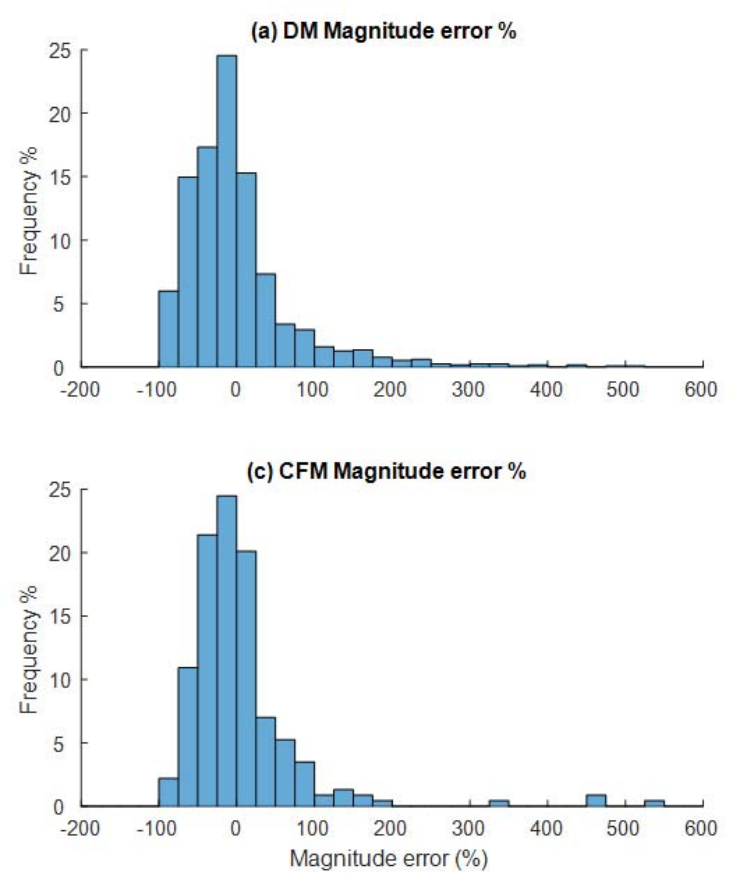
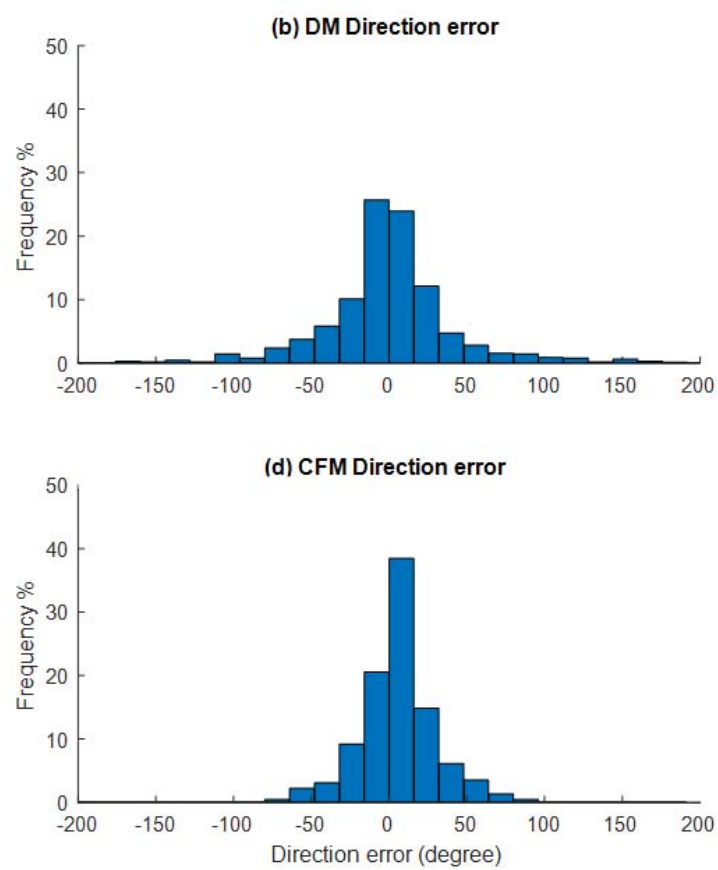

Figure 8. The distribution of errors calculated at each step for both of the steering methods.

378 The error between the predicted (selected) and the actual (real) values is presented, where

379 the positive values indicate greater magnitudes and more clockwise direction in the actual

380 displacement. (b,d): CFM shows a smaller standard deviation in direction error (24 and 41

381 degrees for CFM and DM respectively), likely due to there being a measurement step

382 associated with each manipulation step (compared to the DM that relies on measurements

383 from previous steps). (a,c) The magnitude errors are grouped around close to zero mean,

384 and have similar distributions. The plots are formed from 1190 and 229 data points for the DM and CFM methods respectively.

In Figure $8(b, d)$, the CFM shows less direction error than DM, which is anticipated as it makes measurements more often. Both methods are similar in terms of magnitude errors Figure $8(a, c)$ where the mean and standard deviation magnitude errors are $3.6 \pm 71.6 \%$ and $3.4 \pm$ 
391 from the following causes:

392 A) Timing: variable latencies of $\pm 5.3 \mathrm{~ms}$ in communication between the code and the USB 393 interface caused the frequencies to be applied for periods different to those anticipated.

394 B) Heating effects: Changing temperatures can lead to drift in resonance frequencies that may 395 cause forces to differ between the testing and shifting phases. The DM does less 396 measurement of forces and hence less computation time, which leads to a more sustained application of the manipulating resonances, and hence a higher temperature rise. We typically observe a temperature rises of order 1.7 and $1.0^{\circ} \mathrm{C}$ for DM and CFM respectively during the course of a 1-minute manipulation (measured using a thermocouple on the transducer surface), and thus expect slightly larger errors in the DM from this cause.

401

C) Acoustic field uniformity: The steering algorithm assumes that forces do not vary with small 402 changes of position in order to linearly extrapolate predicted manipulation distances. However there will be small changes (since the manipulation steps are a significant proportion 404 of a wavelength), and will contribute to the errors seen, particularly when the particle displacements approach the scale of force-field variation which is of order $\lambda / 4$ (75 $\mu \mathrm{m}$ at the 406 levitation frequency used here). The force fields will vary between the actual start position 407 of the shift phase and the position at the beginning of the test phase due to the movent 408 caused by each measurement (see Figure 4). This can be mitigated by keep test steps as small 409 as possible (subject to the constraint of also keeping measurement errors resulting from 410 vibrational noise small). The field also varies along the path of the shift phase; this effect is 411 reduced by keeping the shift step size small $(25 \mu \mathrm{m})$. However, there is a trade-off as if this 
413 the testing phase.

414 The contributions of each cause (A,B and C) are harder to quantify, however we observe that 415 over a shifting distance of $25 \mu \mathrm{m}$ forces can often vary by as much as $50 \%$.
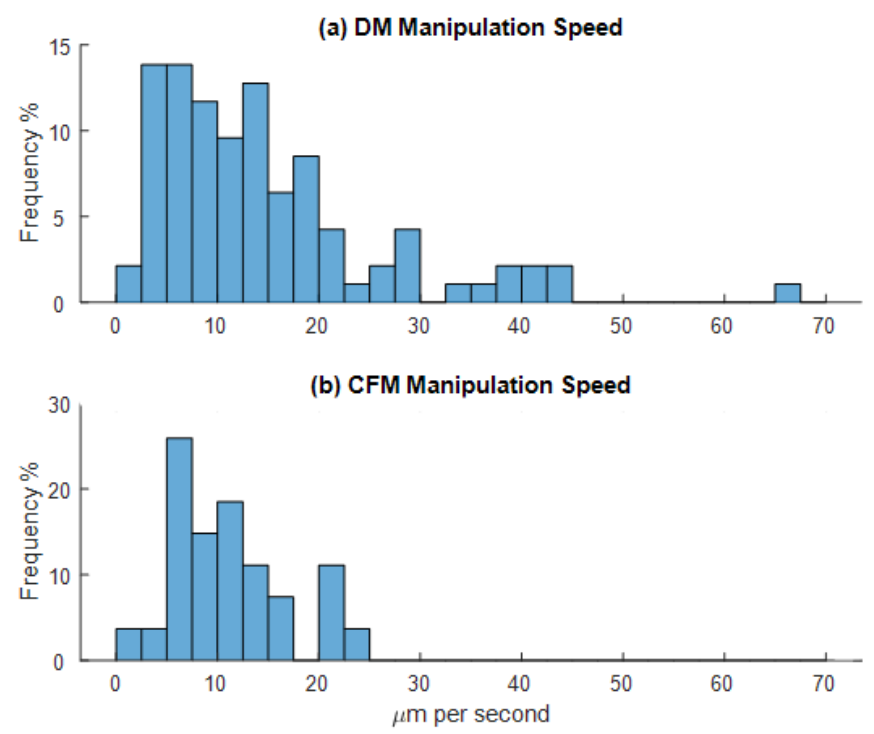

$417 \quad$ Figure 9. The distribution of average manipulation speed for the two different steering

418 methods. Only successful steering legs are included in the generation of these histograms.

419 The average manipulation speed over the course of a leg (from initial position to target) for 420 the two different steering methods have mean values of $15 \pm 11.5$ and $11.2 \pm 5.6 \mu \mathrm{m} / \mathrm{seconds}$

421 for the DM and CFM respectively. The CFM is typically slower than the DM, as a result of it

422 measuring forces at each step. Graphs formed from 94 and 27 data points respectively.

424 Figure 9 compares the average manipulation velocity of the two methods. The reported value 425 is the average velocity that would be required if the manipulation path were straight (i.e. the 426 total straight distance between leg start and target divided by total manipulation time), and 427 does not reflect the average speed over the actual, indirect path that is taken. It can be seen 
that the DM is typically faster than the CFM, which is likely to be a result of the DM measuring

429 forces less often.

430 In order to measure the forces available at each step without unduly disturbing the particle

431 trajectory, the displacements during the test phase are a small number of $\mu \mathrm{m}$ (typically 5-10

$432 \mathrm{um}$ ), which in the presence of the vibrational noise (typically $\pm 1 \mu \mathrm{m}$ ) leads to reasonably high

433 errors between the predicted and actual particle displacements at each shift step. Despite

434 this, the method is robust and able to successfully bring back tracked particles to the direction

435 of the intended target trajectory in around $96 \%$ of legs. The 3D printed and laser cut

436 microscope design that was employed in this study is more sensitive to such vibrations

437 compared to conventional microscopes. We suspect that with lower vibrational noise, it

438 would be possible to achieve more precise and quicker manipulation as smaller, more precise

439 measurement steps could be taken. Another key way to enhance manipulation speed would

440 be to reduce latencies in the system, which could be achieved by using a real-time operating

441 system, I/O hardware with lower latencies, and a camera operating with lower exposure

442 times and faster readout.

443 We also note that more sophisticated, model based algorithms could be employed to

444 significantly enhance manipulation accuracy and speed. For example the acoustic force fields

445 derive from periodic potential landscapes; prior knowledge of the typical wavelength scales

446 of these landscapes coupled with prediction based on stored values from prior locations

447 would give the possibility of reduced testing (through informed prediction), reducing both the

448 number of forces tested and how often they are tested.

449 Observation of the forces associated with each mode under microscopy suggests that the 450 lateral forces associated with each mode primarily result from radiation forces as the forces 
tend act towards points rather than display significant vorticity. However it is a useful feature

452 of the approach taken here that the source of the forces on the particles is not important

453 from the point of view of the control algorithm, as it progresses by measuring the total force

454 from all sources at each measurement step.

455 Armani et al. ${ }^{25}$, demonstrate that in a fluidic system with $\mathrm{n}$ degrees of freedom it is possible

456 to manipulate $(n-1)$ particles independently, as also demonstrated by Zhou et al. ${ }^{26}$. In the

457 current device some additional forces have been obtained through the multiple PZT areas

458 that can be independently actuated, however the available forces are still limited, and the

459 existing forces overlap in terms of directions they offer. We envisage that with more

460 attention to device design it would be possible to create more degrees of freedom in an

461 acoustic device, and hence manipulate more than just one particle at a time.

\section{VIII. CONCLUSIONS}

463 This paper has presented a steering algorithm which uses lateral acoustic radiation forces to control the position of micro particles. It uses feedback based on a microscope imaging system to achieve closed loop control/steering. The successful steering legs have delivered the tracked particles to within $10 \mu \mathrm{m}$ of the target. The field of view is a natural limitation of the current implementation, but at $0.6 \times 0.5 \mathrm{~mm}$ is larger than many comparable techniques.

468 Two variations, labelled the combined forces method (CFM) and the direct method (DM), have been explored, with trade-offs for more accurate paths vs enhanced speed respectively.

470 The methods do not rely on a prior knowledge of the distribution of acoustic forces within the

471 device, but instead measures the forces dynamically during the manipulation leg. Hence, the 472 methods presented are applicable to a wide range of devices and acoustic field structures. 
473 We believe that increased precision and manipulation speed could be achieved beyond this 474 proof of principle study. More sophisticated and predictive algorithms could make better use

475 of the available data to better actuate the device, and we also anticipate designs which limit 476 disturbing temperature variations through wither active cooling, or alternative acoustic 477 design. We also note the device presented here has a rather limited number of resonances 478 (hence different forces) available to the algorithm and that with an alternative device design 479 this could be improved. Manipulating multiple particles is also a possibility in a device with 480 more available resonances.

\section{ACKNOWLEDGEMENTS}

482 Zaid Shaglwf gratefully acknowledges the support of the Ministry of Higher Education and 483 Scientific Research, Libya, for scholarship funding. Peter Glynne-Jones thanks the EPSRC for support from fellowship EP/L025035/1.

Data used to produce the figures in this paper is openly available from the University of Southampton repository at https://doi.org/10.5258/SOTON/D0802

\section{REFERENCES}

$490{ }^{1}$ H. Bruus, J. Dual, J. Hawkes, M. Hill, T. Laurell, J. Nilsson, S. Radel, S. Sadhal, and M. Wiklund,

491 "Forthcoming Lab on a Chip tutorial series on acoustofluidics: Acoustofluidics-exploiting ultrasonic 492 standing wave forces and acoustic streaming in microfluidic systems for cell and particle 493 manipulation", Lab on a Chip (2011). 
${ }^{2}$ B. W. Drinkwater, "Dynamic-field devices for the ultrasonic manipulation of microparticles", Lab on a

495

496

497

498

499

500

501

502

503

504

505

506

507

508

509

510

511

512

513

514

515

516

517

518 Chip 16, 2360-2375 (2016).

${ }^{3}$ S. Gupta, D. L. Feke, and I. Manaszloczower, "Fractionation of Mixed Particulate Solids According to Compressibility Using Ultrasonic Standing-Wave Fields", Chemical Engineering Science 50, 3275-3284 (1995).

4J. J. Hawkes and W. T. Coakley, "Force field particle filter, combining ultrasound standing waves and laminar flow", Sensors and Actuators B-Chemical 75, 213-222 (2001).

${ }^{5}$ S. Oberti, A. Neild, and J. Dual, "Manipulation of micrometer sized particles within a micromachined fluidic device to form two-dimensional patterns using ultrasound.", Journal of the Acoustical Society of America 121, 778-785 (2007).

${ }^{6} \mathrm{M}$. Wiklund, O. Manneberg, B. Vanherberghen, and B. Onfelt, "Flow-free transport of cells in microchannels by frequency-modulated ultrasound", Lab on a Chip 9, 833-837 (2009).

${ }^{7}$ C. D. Wood, J. E. Cunningham, R. O'Rorke, C. Walti, E. H. Linfield, A. G. Davies, and S. D. Evans, "Formation and manipulation of two-dimensional arrays of micron-scale particles in microfluidic systems by surface acoustic waves", Applied Physics Letters 94, 054101 (2009).

${ }^{8}$ X. Y. Ding, S. C. S. Lin, B. Kiraly, H. J. Yue, S. X. Li, I. K. Chiang, J. J. Shi, S. J. Benkovic, and T. J. Huang, "On-chip manipulation of single microparticles, cells, and organisms using surface acoustic waves", Proceedings of the National Academy of Sciences of the United States of America 109, 11105-11109 (2012).

${ }^{9}$ P. Glynne-Jones, R. J. Boltryk, N. R. Harris, A. W. J. Cranny, and M. Hill, "Mode-switching: A new technique for electronically varying the agglomeration position in an acoustic particle manipulator", Ultrasonics 50, 68-75 (2010).

${ }^{10}$ J. R. Wu, "Acoustical Tweezers", Journal of the Acoustical Society of America 89, 2140-2143 (1991).

${ }^{11}$ J. Lee, S. Y. Teh, A. Lee, H. H. Kim, C. Lee, and K. K. Shung, "Single beam acoustic trapping", Applied Physics Letters 95, 073701 (2009). 
$519{ }^{12} \mathrm{~J}$. Lee, C. Lee, H. H. Kim, A. Jakob, R. Lemor, S. Y. Teh, A. Lee, and K. K. Shung, "Targeted cell

520 immobilization by ultrasound microbeam", Biotechnol Bioeng 108, 1643-1650 (2011).

$521{ }^{13}$ T. Kozuka, T. Tuziuti, H. Mitome, T. Fukuda, and F. Arai, "Control of position of a particle using a standing wave field generated by crossing sound beams", 1998 IEEE Ultrasonics Symposium Proceedings, Vols 1 and 2, 657-660 (1998).

${ }^{14}$ A. L. Bernassau, P. Glynne-Jones, F. Gesellchen, M. Riehle, M. Hill, and D. R. S. Cumming, "Controlling acoustic streaming in an ultrasonic heptagonal tweezers with application to cell manipulation", Ultrasonics 54, 268-274 (2014).

"Manipulation of microparticles using phase-controllable ultrasonic standing waves", Journal of the Acoustical Society of America 128, EL195-199 (2010).

$530{ }^{16}$ L. Meng, F. Cai, J. Chen, L. Niu, Y. Li, J. Wu, and H. Zheng, "Precise and programmable manipulation

531 of microbubbles by two-dimensional standing surface acoustic waves", Applied Physics Letters 100,

$532 \quad 173701-173704(2012)$.

${ }^{17}$ C. R. P. Courtney, C. E. M. Demore, H. X. Wu, A. Grinenko, P. D. Wilcox, S. Cochran, and B. W.

Drinkwater, "Independent trapping and manipulation of microparticles using dexterous acoustic tweezers", Applied Physics Letters 104, 154103 (2014).

${ }^{18}$ A. Riaud, M. Baudoin, O. B. Matar, L. Becerra, and J. L. Thomas, "Selective Manipulation of (2017).

$539{ }^{19}$ A. Marzo, S. A. Seah, B. W. Drinkwater, D. R. Sahoo, B. Long, and S. Subramanian, "Holographic 540 acoustic elements for manipulation of levitated objects", Nature Communications 6, 8661 (2015).

$541{ }^{20} \mathrm{~T}$. Kozuka, T. Tuziuti, H. Mitome, and T. Fukuda, "Control of a standing wave field using a line-focused 542 transducer for two-dimensional manipulation of particles", Japanese Journal Of Applied Physics Part 543 1-Regular Papers Short Notes \& Review Papers 37, 2974-2978 (1998). 

12554 (2013).

${ }^{22}$ U. S. Jonnalagadda, M. Hill, W. Messaoudi, R. B. Cook, R. O. C. Oreffo, P. Glynne-Jones, and R. S. Tare,

${ }^{23}$ P. Glynne-Jones, R. J. Boltryk, and M. Hill, "Acoustofluidics 9: Modelling and applications of planar resonant devices for acoustic particle manipulation", Lab on a Chip 12, 1417-1426 (2012). manipulation of particles in planar acoustic resonator", IEEE Trans Ultrason Ferroelectr Freq Control 59, 1258-1266 (2012).

${ }^{25}$ M. D. Armani, S. V. Chaudhary, R. Probst, and B. Shapiro, "Using feedback control of microflows to independently steer multiple particles", Journal of Microelectromechanical Systems 15, 945-956 (2006).

${ }^{26} \mathrm{Q}$. Zhou, V. Sariola, K. Latifi, and V. Liimatainen, "Controlling the motion of multiple objects on a Chladni plate", Nature Communications 7, 12764 (2016).

${ }^{27}$ Microscale acoustofluidics. Editors: Thomas Laurell, Andreas Lenshof. Publisherd by the Royal Society of Chemistry) ISBN 978-1-84973-671-8 (2015). Physics Doklady, Vol. 6 (1962) 773. standing wave fields", Journal of the Acoustical Society of America 77, 928-945 (1985). resonant devices for acoustic particle manipulation", Lab Chip 12, 1417-1426 (2012).

$568{ }^{31}$ M. Wiklund, R. Green, and M. Ohlin, "Acoustofluidics 14: Applications of acoustic streaming in microfluidic devices", Lab on a Chip 12, 2438-2451 (2012). 
$570 \quad{ }^{32}$ B. Hammarstrom, M. Evander, H. Barbeau, M. Bruzelius, J. Larsson, T. Laurell, and J. Nilsson, "Non-

571 contact acoustic cell trapping in disposable glass capillaries", Lab on a Chip 10, 2251-2257 (2010).

$572{ }^{33} \mathrm{P}$. Hahn and J. Dual, "A numerically efficient damping model for acoustic resonances in microfluidic 573 cavities", Physics of Fluids 27, 062005 (2015).

$574{ }^{34}$ J. F. Spengler, M. Jekel, K. T. Christensen, R. J. Adrian, J. J. Hawkes, and W. T. Coakley, "Observation 575 of yeast cell movement and aggregation in a small-scale MHz-ultrasonic standing wave field", 576 Bioseparation 9, 329-341 (2001).

$577{ }^{35}$ See supplementary material at [URL will be inserted by AIP] for SuppPubmm3.avi, an exanple video 578 of a square trajectory with the DM method.

$579{ }^{36}$ See supplementary material at [URL will be inserted by AIP] for SuppPubmm1.avi, an exanple video 580 of the CFM method.

$581{ }^{37}$ See supplementary material at [URL will be inserted by AIP] for SuppPubmm2.avi, an exanple video 582 of the DM method. 